\title{
Theater als didaktisches Mittel für den Spracherwerb Gedanken zur zweisprachigen Textdramatisierung
}

MorToN MüssTer

Universidad de Extremadura

Eberhard Karls Universität Tübingen

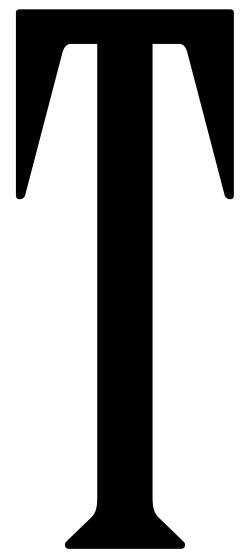

heaterarbeit ist ein sehr komplexes und zeitintensives Unterfangen, das aber auch sehr gewinnbringend sein und interdisziplinär genutzt werden kann, was Schewe (2007) sehr ausführlich dargelegt hat. Der hier angeführte Erfahrungsbericht beruht aber auf einer zweisprachigen Theaterarbeit im Bereich Deutsch als Fremdspra-

che an der Universidad de Extremadura. Es stand also vor allem die gegenseitige Bereicherung von Theater und Zweitspracherwerb im Vordergrund. Welche Ziele und Vorteile ein solches Projekt im Allgemeinen und für Zweisprachigkeit im Speziellen haben kann, sei nachfolgend knapp zusammengefasst.

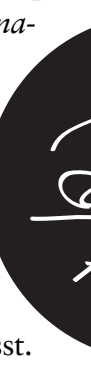

Rein politisch argumentiert dient ein Theaterprojekt auf Deutsch an einer ausländischen Universität der Werbung. Denn mit einer Theateraufführung wird eine mediale Aufmerksamkeit erzeugt, die dem betreffenden Studiengang zugutekommen kann. Es wird zudem suggeriert, dass der Studiengang ein gutes Kursangebot anbietet, indem es neben den Pflichtkursen auch ein kulturelles Zusatzangebot bereitstellt. Der Faktor Zweisprachigkeit ist dabei nicht zu vernachlässigen. Denn durch ihn gelingt es, an eine größere Zielgruppe zu gelangen. Auch Publikum und Studenten mit geringen oder keinen Deutschkennt- nissen wird so der Zugang zu der Materie nicht nur ermöglicht, sie werden sogar motiviert, weil die zweisprachige Inszenierung ihnen ermöglicht, das dargestellte literarische Werk zu verstehen, ohne des Deutschen mächtig zu sein. Damit kann man die mentale Einstiegshürde, Deutsch zu lernen, senken helfen. Gerade in Spanien gilt das Deutsche als sehr schwierig, was viele eigentlich Interessierte daran hindert, die Sprache zu lernen. Mit einer erfolgreichen zweisprachigen Inszenierung schafft man aber im Publikum, das mehr verstanden hat als es zunächst erwartet hatte, ein Erfolgserlebnis, welches womöglich doch zum Deutschlernen motiviert. Ein wesentlicher weiterer Vorteil der zweisprachigen Inszenierung liegt darin, dass man das zu bearbeitende Drama ungekürzt und quasi im Original aufführen kann. Damit dient es der Vermittlung der deutschsprachigen Literatur und Kultur in großem Maße und kann auch hier die Zielgruppe motivieren, sich weiter mit deutscher Sprache, Literatur und Kultur auseinanderzusetzen.

Von diesen werbestrategischen und sprachenpolitischen Faktoren abgesehen bietet die fremdsprachige bzw. zweisprachige Theaterarbeit noch weitere Vorteile, die es auch zu beachten gilt. Gerade im Kontext einer philologischen Bildung stellt es eine Bereicherung dar, wenn neben den gelernten Dramen-

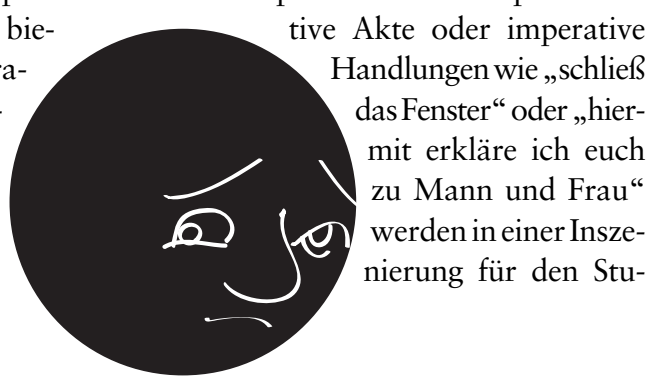




\section{Abstract}

In dem vorliegenden Beitrag wird versucht, dem Leser einen Einblick in die Abläufe eines im Kontext des Fremdsprachenunterrichts stehenden zweisprachigen Theaterseminars zu geben. Dabei werden organisatorische, didaktische, dramaturgische und philologische Aspekte berücksichtigt. Es sei skizziert, worum es in einem solchen Seminar geht, wobei Thomas Bernhards Drama Die Jagdgesellschaft beispielhaft für eine zweisprachige Theaterarbeit im Mittelpunkt der Analyse steht. Die im Vordergrund stehende zweisprachige Konzeption hat zahlreiche Auswirkungen auf theoretische und auch praktische Aspekte des Theaters, sowie auf deren Didaktik.

Schlüsselwörter: Deutsch als Fremdsprache, Theater, Didaktik, Thomas Bernhard

\section{Resumen}

El presente artículo se propone dar una visión cronológica y desde dentro de un taller de teatro bilingüe, organizado en el marco de las clases de alemán como lengua extranjera. Se contemplan aspectos organizativos, didácticos, dramatúrgicos y filológicos del trabajo con la obra La partida de caza de Thomas Bernhard. El enfoque bilingüe del montaje tiene muchas implicaciones para aspectos prácticos u teóricos del teatro y su didáctica.

Palabras clave: Alemán como lengua extranjera, teatro, didáctica, Thomas Bernhard. dierenden verständlicher und damit leichter lernbar. Weitere symbiotische Effekte zwischen dramapädagogischer Arbeit und Spracherwerb sind in der Wortschatzarbeit (Benson 2001), im erheblichen Redeanteil des Sprachschülers in der

Fremdsprache in den Proben und der Inszenierung, Stichwort Kontaktzeit (McDonell 1992), und im Abbau der Fiktivität der Fremdsprache (Hallet 2010) zu sehen; mit dem Gebrauch der Fremdsprache in der Inszenierung gewinnt ihr Gebrauch einen realen Nutzen, was zu einem effizienterem Lernen führen kann. Dies schließt an die Ausführungen zu dem Nutzen performativer und symbolischer Elemente im Fremdsprachenerwerb an. Die Wortschatzarbeit schließlich geschieht auf vielfältige Weise, zum einen wird der Wortschatz mit dem zu dramatisierenden Text erweitert, zum anderen werden auch Fachbegriffe aus der Theaterarbeit in den Wortschatz eingebracht, da es neben der Textarbeit auch eine Arbeit mit dem Text als Vorbereitung auf eine Inszenierung gibt. Ein bedeutender Vorteil dieser Wortschatzarbeit besteht in seiner gerade nicht theoretischen Natur, was dem Spracherwerb den fiktiven Rahmen nimmt und ihn real bedeutsam werden lässt.

Theaterarbeit bringt zudem die Erwerbsmöglichkeit einer ganzen Reihe von Schlüsselqualifikationen mit sich, was in der heutigen Zeit immer wichtiger wird. So plädiert Byram (1999) für den intercultural speaker, der das unerreichbare Ideal des

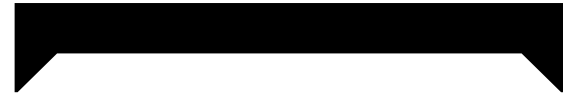

Ein bedeutender Vorteil dieser

Wortschatzarbeit besteht in seiner gerade nicht

theoretischen Natur, was dem Spracherwerb

den fiktiven Rahmen nimmt und ihn real

bedeutsam werden lässt.

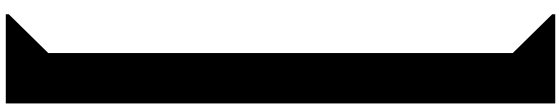

native speakers ablösen soll. Neben der Frustration, das Niveau eines natives nicht erreichen zu können, bietet der intercultural speaker den Vorteil, Zusammenhänge zwischen Sprachen und Kulturen zu erkennen, statt dem Ideal der Perfektion hinterherzurennen. Zu den Schlüsselqualifikationen, die erworben werden können, gehört aber neben der Förderung der sprachlichen Kompetenz auch das selbstbewusste Auftreten vor Publikum, das disziplinierte, solidarische Arbeiten im Team und die kreative Arbeit und Improvisationsfähigkeit.

\section{Schwierigkeiten}

Bei der Umsetzung der oben genannten Ziele innerhalb eines solchen Theaterprojekts stößt man jedoch auf zahlreiche Schwierigkeiten. So ist etwa der Faktor Zeit sehr problematisch; den Teilnehmern ist innerhalb sehr kurzer Zeit ein Textverständnis zu vermitteln. Außerdem müssen sie in demselben Zeitraum erste schauspielerische Fähigkeiten und Techniken erlernen, muss man doch davon ausgehen, dass viele zum ersten $\mathrm{Mal}$ auf der Bühne stehen werden. Mit in diesen Faktor Zeit fällt auch das Textlernen, was am Anfang die meisten Schwierigkeiten bereiten wird, aber die Arbeitsbasis bedeutet und daher von höchster Wichtigkeit ist. Trotz zahlreicher Vorteile, die eine zweisprachige Inszenierung

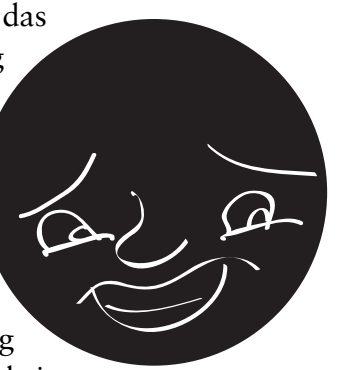
mit sich bringt, stellt sie doch beim

Lernen des Theatertextes eine enorme Schwierigkeit dar. Je nach Konzeption der zweisprachigen Inszenierung können entweder einzelne Personen eine spezifische Sprache ${ }^{1}$ vertreten - dies bereitet Probleme bei den Einsätzen — oder Sprache wechselt aufgrund inhaltlicher Motivationen ab (dies ist der Fall bei der Inszenierung der Jagdgesellschaft und wird weiter unten näher erläutert), was dazu führt, dass ein Schauspieler einen durch Zweisprachigkeit gekennzeichneten, sehr heterogenen Text erlernen muss. In beiden Fällen erzeption das Textlernen. So ist es eine prioritäre Aufgabe, die Teilnehmer in Disziplin zu schulen, ohne sie dabei zu demotivieren oder zu überfordern; die Freude an der Theaterarbeit muss 
essenziell sein! Am Ende dieser intensiven Phase der Körperschulung und des Textlernens sollen die gewonnenen Erkenntnisse umgesetzt werden. Das zeigt sich optimalerweise in einer geglückten Aufführungssequenz, obwohl man, was ganz normal ist, viel zu wenig Zeit zur Vorbereitung gehabt haben wird.

Viele von den Kursteilnehmern sind keinePhilologiestudenten. Man muss auch nicht Philologe sein, um Theater spielen zu können. Es ist dann aber schwieriger, am Textverständnis $\mathrm{zu}$ arbeiten und theoretische Grundlagen ansatzweise zu ver-

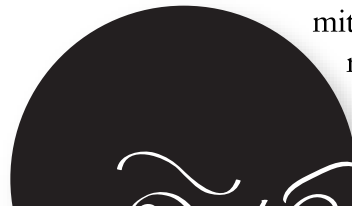
itteln. Sowohl eine theoretische Mindestgrundlage, als auch ein philologisches Textverständ6 ก 2 nis sind zumindest für ein solches Seminar wünschenswert, findet es doch im Rahmen des philologischen Fremdsprachenerwerbs statt. Für die Schauspieler ist es viel leichter, mit Texten umzugehen, die sie verstehen; mit denen sie etwas anfangen können. Nur ein Beispiel: In Thomas Bernhards Theaterstücken findet sich eine unkonventionelle Syntax, die man eher dem Bereich der Lyrik als der Prosa zuordnen würde. In der sich - frei nach Jakobson - die paradigmatische über die syntagmatische Ebene erhebt. Es handelt sich um unvollständige, aneinander gereihte Wörter. Der Begriff der Phrasen trifft nicht zu, handelt es sich zwar um nur anscheinend arbiträr gebildete Wortreihen, diese folgen aber nicht grammatisch-syntaktischen Prinzipien. Diese Wörter ordnen sich offenbar nach zwei Prinzipien: (i) Assoziationen und (ii) Rhythmus. Der Assoziationsaspekt steht für die inhaltliche Seite. Anhand bestimmter Signalwörter oder Wortkombinationen assoziiert der Leser oder Zuschauer, in jedem Fall der Rezipient Bilder bzw. Inhalte in seinem Bewußtsein. Es enstehen Assoziationsketten, die einen roten Faden im Drama bilden.

Die rhythmische Ebene funktioniert dazu parallel. Oft wird in Bernhards Werk eine große Musikalität gesehen, die man mit barocken Kompositionsprinzipien vergleichen könnte, wie etwa dem Prinzip derFuge. Durchauskann man gelegentlich in der Rhythmik des Textes auch einen Wal-

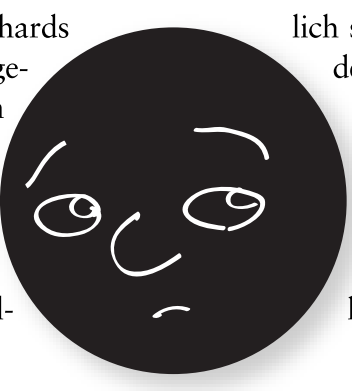
dend), der aus der intendierten denen zu lernen. Und selbst wenn das Auswendiglernen in diesem Fall geglückt sein sollte, wird es den zukünftigen Schauspieler vor eine fast unüberbrückbare Schwierigkeit stellen, den gelernten Text darzu-

zertakt oder Tanz erahnen. Es entsteht ein Rhythmus (sich selbstverständlich aus unterschiedlichen Längen und Pausen bilSetzung der Wörter - die Reihenfolge ist demnach sensibel - entsteht. Dabei Wechseln sich Wiederholungen, Alternanzen, bestimmte Motive, etc. ab. Bei der Sprache Bernhards handelt es sich um eine sensible Form-Inhalt-Komposition, die in ihrer Wirkung vor allem eine ästhetische ist, d.h. um ihrer selbst

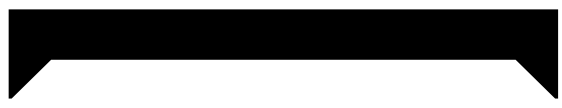

Off wird in Bernhards Werk eine große Musikalität gesehen, die man mit barocken Kompositionsprinzipien vergleichen könnte, wie etwa dem Prinzip der Fuge. Durchaus kann man ge-

legentlich in der Rhythmik des Textes auch ei-

\section{nen Walzertakt oder Tanz erahnen.}

Willen im Sinne der Selbstreferenzialität.

Diese Konkretisierung soll verdeutlichen helfen, warum es für den Schauspieler von großem Nutzen ist, eine theoretische Grundlage zu besitzen. Mit einer rein hermeneutischen und auf konventionelle Inhalte gerichteten Textanalyse würde er recht hilflos vor dem Drama stehen. Dass dieses zu Demotivation führen kann, ist nur ein Nebeneffekt. Einen nicht verstandenen Text auswendig zu lernen, ist deuth schwieriger, als einen verstansame Wissen von der Sache kann jeder das Resultat nämlich in Abweichung vom im Bewußtsein vorgestellten Original beurteilen. Dieses Unterfangen wäre auch zum Scheitern verurteilt, ginge es beim Theater tatsächlich darum, eine absolute Rea- 
lität zu imitieren. Vielmehr geht es aber beim Theater um das Prinzip der Wahrscheinlichkeit und der Andeutungen. Die Frage nach dem so könnte es doch sein oder passieren tritt in den Vordergrund.

Aber selbst, wenn man das alles verinnerlicht und akzeptiert hat, bleibt doch das Problem des eigenen Körpers. Selbst wenn man die Theorie kennt und sich nicht scheut, können die meisten die erworbene Theorie nicht in die Praxis umsetzen. Der fehlende Zugang zum eigenen Körper, mit anderen Worten, das Nicht-Bewußtsein vom eigenen Körper und dessen fehlende oder mangelhafte Kontrolle behindern das Theaterspiel massiv. Oft führen wir ganz absurde Bewegungen unterschiedlichster Körperglieder ganz unbewußt in den unterschiedlichsten Situationen aus, sei es beispielsweise beim Reden oder beim Essen, aus Nervosität oder aus Gewohnheit. Dass man den eigenen Körper jedoch nicht beherrscht, merkt man oft schon, wenn man versucht, sich beim Vortragen einer Textpassage überhaupt nicht zu bewegen. Der Kopf, die Hände, die Schultern, Arme, Beine, Füße, die Augen - wie auch immer das Nicht-Bewegen scheint ein unmögliches Unterfangen zu sein. Daher gehört die Schulung der Körperkontrolle zu den zentralen, zeitaufwendigen Aufgaben in einem Theaterseminar.

Parallel dazu sei auch die sprachliche Expressionskompetenz erwähnt. Denn das Ziel liegt dort zwischen zwei Extremen: zum einen die leiernde Rezitation von gelerntem Text, was nach wenigen Sekunden zu Ermüdung und Desinteresse des Publikums führen würde und zum anderen eine ungezügelte, schlecht artikulierte, jedoch im wörtlichen Sinne natürliche Umgangssprache. Um nochmals auf eines der Hauptprinzipien des Theater zu erinnern; es geht nicht um die reale, mimetische Abbildung einer absoluten Realität, sondern vielmehr um die Darstellung einer möglichen, wahrscheinlichen Situation auf der Bühne. Dieses ist aber mit den beiden eben genannten Extremen bei der sprachlichen Expression nicht zu erreichen. Es bedarf sowohl einer gewissen Natürlichkeit als auch einer sehr deutlichen, distinguierten Theatersprache.

Ein weiteres, sehr bekanntes Problem ist das so genannte Lampenfieber. Im Prinzip haben wir das eben schon unter dem Punkt psychische Hemmungen angespro- chen. Es handelt sich aber bei dem Lampenfieber um einen speziellen Fall, der nicht die Zeit des Seminars, die Übungszeit, störend betrifft, sondern erst kurz vor den Aufführungen, just in time vor der Premiere auftritt: die plötzlich auftauchende Angst davor, auf der Bühne zu stehen und vor Publikum zu spielen, womöglich dabei zu versagen. In den meisten Fällen legt sich diese Befangenheit mit den ersten ausgeführten Bewegungen und geäußerten Worten wieder. In Ausnahmen kann das Lampenfieber aber auch eine übermäßige

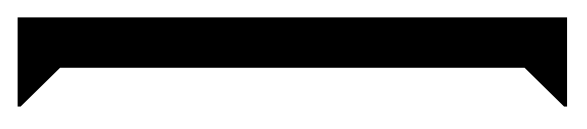

Der fehlende Zugang zum eigenen Körper, mit anderen Worten, das Nicht-Bewußstsein vom eigenen Körper und dessen fehlende oder mangelhafte Kontrolle behindern das

\section{Theaterspiel massi}

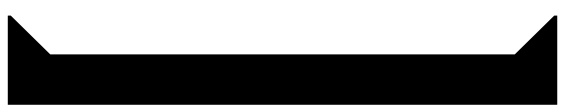

Nervosität bewirken, die zu einer Paralysierung von Körper und Sprache führen würde. Passiert so etwas auf der Bühne, versteht man, warum ein Amateur-Ensemble eine funktionierende Gruppendynamik besitzen muss. Denn alleine wird man eine solche Situation kaum gesichtswahrend überwinden können. Aber aus Erfahrung läßt sich sagen, dass solche, wirklich seltenen Situationen immer gerettet werden. Zwei weitere Kompetenzen müssen also in einem Theaterseminar geschult werden: mit Gruppenarbeit und

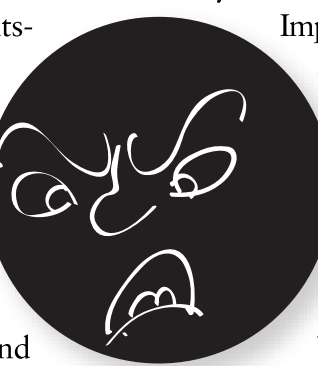
Im zweiten Bild des Dramas sehen wir auf der Bühne eine ältere Frau und ihre

Tochter, bereits eine junge Frau, unverheiratet, die jüngste der Geschwister, dazu bestimmt, die Mutter bis zu ihrem Ableben zu pflegen und selbst nicht heiraten zu dürfen. Die Mutter wird an die Tür gebeten — von der Magd einer Nachbarin— selbstverständlich als Ablenkungsmaßnahme. Dadurch ist die jüngste Tochter einen Augenblick unbewacht und ein Nachbarskind kann einen Brief vom Liebhaber, der die Magd bestochen hat, aushändigen. Zusammen mit dem Brief soll das Nachbarskind eine mündliche Botschaft überbringen. Vor plötzlich auftretender Aufregung (Lampenfieber) vergisst das Kind fast den Text und liest ihn rasch vor dem Auftritt nochmals, um das Kurzzeitgedächtnis zu aktivieren. Dadurch lenkt sich die Konzentration auf die Textmitteilung. Das Kind, kaum die Bühne betreten, will in ängstlicher Aufregung nur noch die Textpassage vortragen und vergisst, dass der Schlüssel der Situation aber der Brief ist. In diesem aufgeregten Eifer ist der Textdrang des Kindes kaum zu stoppen. Aber die Tochter kann die Situation noch retten und fragt: „Aber sag einmal, hat dir Leo keinen Brief für mich mitgegeben? “ und bekommt den Brief vom Kind ausgehändigt. Umgekehrt hätte das Nachbarskind auch pflichtbesessen den Brief übergeben aber den Text vergessen haben können. In dem Fall hätte die Tochter fragen müssen: „Und hat Leo noch etwas gesagt, als er dir den Brief gab?“. Von der Banalität dieses Beispiels abgesehen, läßt sich doch verdeutlichen, wie wichtig eine Emanzipation von der stupiden Textrezitation hin zu einer improvisationsfähigen, eigene Nuancen setzenden Gruppendynamik ist. Denn Gruppendynamik und Improvisationsvermögen sind die Basis für das geheimnisvoll anmutende Eigenleben des Theaterspiels. Gerade dieses charakteristische Sich-Verselbstständigen des Textes grenzt die Dramatik so deutlich von der Narrativik und der Lyrik ab. Wie sich bisher gezeigt hat, die Improvisationsfähigkeit. Zur Verdeutlichung ein weiteres Beispiel aus einem Werk, das die Aufname in den Kanon der Weltliteratur um ein Haar verfehlt hat: kommt es beim Theaterspiel auf zahlreiche Details und Fähigkeiten an. Gruppenarbeit ist gefordert. Ohne einen großen Zeitund Arbeitsaufwand ist ein Theaterprojekt 
gar nicht zu realisieren. Das alles ist nur mit Disziplin und Lust an der Sache zu bewältigen; eine weitere Schwierigkeit des Theaterseminars. Ist es doch keine einfache Aufgabe, einen Studenten bzw. eine ganze Horde Studenten dazu zu bringen, freiwillig und zum unentgeltlichen Eigenzweck diszipliniert, zum Teil selbstständig, in einer Gruppe zu arbeiten. Denn ohne Disziplin ist eine Amateurgruppe gar nicht zu bändigen, will man das Ziel einer Aufführung erreichen (von der Qualität einer solchen Bühnenshow zunächstganzabgesehen). Und damit nicht genug, schließlich verfolgt man im Allgemeinen auch noch künstlerische Absichten, die weit über eine simple Textrezitation hinausgehen. Doch muss man mit dem Allheilmittel Disziplin vorsichtig und sparsam umgehen. Die Lust am Theaterspiel ist genauso wichtig, wie eine rein technisch (durch erfolgreiche Gruppendisziplin möglich gewordene) geglückte Aufführung; weder das Publikum noch das Ensemble hätten Freude daran, wenn in der Folge anhaltender Demotivation, in Zeiten unermüdlicher Disziplinierungsversuche das Ensemble nicht schon auseinander gebrochen wäre. Der Faktor Motivation ist also einerseits während der Probenzeit - damit wie schon gesagt ein Durchhalten der Mannschaft gewährleistet ist - als auch andererseits während der Aufführungen sehr wich-

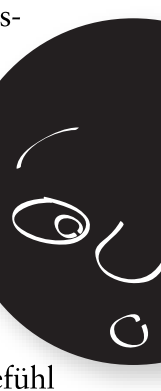
tig. Um sowohl ein Gruppengefühl zu stärken als auch Motivation und Freude zu fördern, sind gemeinsame Aktivitäten außerhalb der Proben von Vorteil. Denn die Freude an der Darstellung verleiht den Aufführungen einen entscheidenden Mehrwert, der das Spiel lebendiger und expressiver macht.

\section{Organisation}

An diese Schilderung der Ziele und Probleme anschließend, werde ich eine Art Arbeitsplan umreißen. Dieser Arbeitsplan soll einen Überblick der Prozesse und Teilschritte verschaffen, die von der Auswahl eines Textes ganz am Anfang bis abschließend zu den Aufführungen selbst reicht.

Ganz am Anfang muss es eine Motivation geben, ein Theaterprojekt durchzuführen. Das mag sich trivial anhören. Wer allerdings versucht hat, ein Theaterprojekt zu leiten, weiß, wie wichtig Ideen und Motivation sind. Im besten Fall handelt es sich dabei um eine penetrante, brennende Idee, welche die Seelenruhe quält, bis man sich der Idee endlich annimmt. Dann handelt es sich um eine Leidenschaft, was in der Regel einen späteren Erfolg auf der Bühne garantiert - ein Mindestmaß an Talent, Geduld und Disziplin vorausgesetzt. Natürlich kann es auch andere Motivationen geben, z.B. ein Arbeitsvertrag, aus dem eine Bring-Pflicht hervorgeht. Es kann aber auch eine Mischung daraus sein, dass man beispielsweise regelmäßig ein Projekt durchführen muss oder sollte und dann glücklicherweise auch ständig Ideen entstehen. So eine Symbiose ist ideal. Denn frei nach Brecht muss es immer auch jemanden geben, der dem Weisen die Weisheit entlockt und sie aufschreibt; in diesem Fall die Bringpflicht, welche die Ideen katalysiert.

Wie sehen die nächsten Schritte aus? Das sei hier nur knapp aufgelistet: Teilnehmer verpflichten, Inszenierungsideen konkretisieren, ggfs. Textänderungen durchführen, die Rollen lernen, den Text gemeinsam interpretieren, die Figuren gemeinsam charakterisieren, theoretische Grund6 lagen vermitteln, Körper und Sprache schulen, gegen Ende die Darstellung und Choreographie perfektionieren und an die Inszenierungsidee anpassen. Zudem fallen organisatorische Aufgaben wie Buchhaltung, Werbung, Öffentlichkeitsarbeit und Sponsering an. Es müssen Kostüme, Requisiten und andere Materialien beschafft werden. Wenn man keinen festen Theaterraum hat, müssen zudem die Lokalitäten gesucht, inspiziert und Licht- bzw. Bühnenbildfragen geklärt werden.

Es folgt die Generalprobe. Die Generalprobe zeichnet sich zunächst dadurch aus, dass alles ablaufen soll, als handele es sich um eine Premiere. Es kommt nun darauf an, das Theaterstück als Gruppe und als Individuum verinnerlicht $\mathrm{zu}$ haben. Man muss die darzustellende Situation für den Moment der Generalprobe für sich annehmen und ausfüllen. Man leiht der Dramenfigur für die Zeit der Generalprobe seinen Körper. Dasselbe gilt natürlich für die Premiere und die weiteren Aufführungen. Nur dass fortan Publikum mit im Raum

\section{Zweisprachigkeit in Bernhards Die Jagdgesellschaft}

Zum Schluß einige Bemerkungen zu dem 2008 an der Universidad de Extremadura durchgeführten Theaterprojekt Die Jagdgesellschaft als zweisprachiges Experiment. Es ist keineswegs weit hergeholt oder absurd, das Drama Die Jagdgesellschaft mit spanischen und deutschen Elementen zu versetzen. Dabei ist zum einen Bernhards Zuneigung zur spanischen Kultur und Sprache zu nennen, die er paradoxerweise selbst gar nicht beherrschte. So berichtet Bernhard ungezwungen in einem Interview, dass er es als sehr angenehm empfände in Spanien zu sein, da er -weil er ja der spanischen Sprache nicht mächtig sei- in Spanien das Gefühl habe, die Leute um ihn herum würden ständig nur angenehme Dinge sagen. Das kann man im Kontrast zu seinem verhaßten Österreich sehen, wo er die Vulgaritäten seiner Landsleute gemäß eigener Aussage nicht ertragen konnte. Diese Abneigung geht in fortgeschrittenem Alter so weit, dass Bernhard in seinem Testament vorsieht, die Aufführung seiner Stücke in Österreich nach seinem Ableben verbieten zu lassen.

Zum anderen, und das baut auf dem eben Ausgeführten auf, ist die Zweisprachigkeit gewissermaßen schon im Stück enthalten (dazu auch Münster 2008). Zwar geht es nicht um die Alternanz der Idiome Spanisch und Deutsch, wohl aber um die Anwesenheit sprachlicher Barrieren. Man kann in dem so rhythmisch und symbolisch geprägten Drama das Motiv der unüberwindlichen, menschlichen Nicht-Kommunikation sehen. Und genau das passiert, wenn zwei fremde Sprachen, Spanisch und Deutsch, 
aufeinandertreffen, der Rhythmus und die Melodie wechseln dabei. Ganz zu Beginn des Stücks bedient sich der Autor beispielsweise der französischen und lateinischen Sprache und provoziert damit metrische Wechsel in der Sprache.

Die Figuren im Stück werden in ein Vakuumgeworfen, da man ihnenjeglichen Haltnimmt, Zeit und Raum haben keinen Wert mehr. Das Suchen nach Fixpunkten, das Warten auf etwas, aber was denn eigentlich? protagonisieren

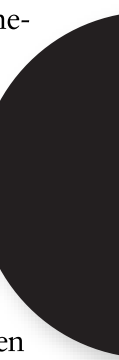
das Spiel. Die in ihrer konventionellen Funktion nicht mehr funktionierende Sprache (Nicht-Kommunikation) dient nur noch der Ästhetik, die sich des Rhythmus', der Musikalität und der assoziativen Symbole bedient. Ein Hinhalten im Nichts, während sich nichts ändert, bis das Nichts am Ende eintrifft. Mit anderen Worten: ein unterhaltsames Festhalten am Nichts; ein autoreferenzielles, dem Selbstzweck dienendes, rein sensualistisches, ästhetisches Erleben.

GENERALIN Dann sitzen Sie/ stumpf/ stumm/ mit herunterhängendem Kopf/ Dann ist es das bis an die Grenze des Verrücktwerdens gehende Schweigen/ nichts

SCHRIFTSTELLER Nichts/ Immer wieder nichts

G Und dann kommen Sie auf die Idee/ Siebzehnundvier zu spielen/ ununterbrochen/tagelang/ und reden nichts/ und gewinnen/ und gewinnen fortwährend/ mit erschreckender Sicherheit/ Und ich verliere/ mit der gleichen/ ununterbrochenen Sicherheit

S Dieses stumpfsinnigste aller Kartenspiele [...]

G Die Zeit überbrücken/ nichts als die Zeit zu überbrücken.

(Bernhard 2005: 179-182)
Fazit

Eine zweisprachige Inszenierung kann Vor- und Nachteile haben, strategisch, didaktisch oder inhaltlich motiviert sein. Es ist in jedem Einzelfall abzuwägen, ob ein zweisprachiges Projekt sinnvoll ist. Zwarkann dies, wie oben ausgeführt, einem ganz neueZielgruppen und andere Lerneffekte ermöglichen. Doch sollte $\curvearrowleft$ man nicht außer Acht lassen, dass ein zweisprachiges Theaterseminar sicher

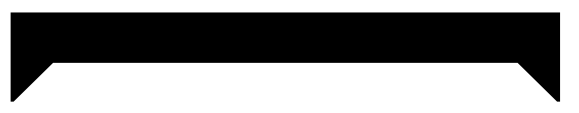

Ein Hinhalten im Nichts, während sich nichts ändert, bis das Nichts am Ende eintrifft. Mit anderen Worten: ein unterhallsames Festhalten am Nichts; ein autoreferenzielles, dem Selbstzweck dienendes, rein sensualistisches, ästhetisches Erleben.

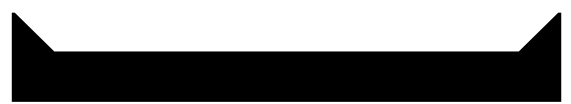

Mehrarbeit, auch Probleme, mit sich bringt und sich nicht jeder Text sich zur zweisprachigen Dramatisierung eignet. So bietet sich etwa die zweisprachigeDramatisierung vor allem aus sprachenpolitischen Gründen und motivationsbedingtan, wenn das Sprachlernen des Deutschen in einer bestimmten Region niedrig ist und man auf die Landessprache angewiesen ist, um ein Zielpublikum zu erreichen. Damit zusammen hängt auch die erwähnte Senkung der Hürde, Deutsch zu lernen, wenn man den Unterricht mit zweisprachigen Konzepten beginnt. Andererseits, und das hat sich in der Inszenierung der Jagdgesellschaft gezeigt, kann es aus philologischen Gründen interessant sein, ein Stück zweisprachig zu inszenieren. Dass dabei nicht jeder Text geeignet ist, steht außer Frage.

Gegen eine zweisprachige Inszenierung oder Unterrichtskonzeption sollte man sich allerdings entscheiden -vorausgesetzt man entscheidet sich nicht aus den oben genannten philologischen Gründen für eine zweisprachige Inszenierungwenn sowohl die Deutschkenntnisse der Studierenden für ein Theaterprojekt ausreichend sind, d.h., dass sie in der Lage sind, eigenverantwortlich den

Text auswendigzu lernen und unter Anleitung zu verstehen und es genug Zielpublikum sowohl für eine potenzielle Aufführung als auch als Teilnehmer für den Kurs zu erwarten sind. Ein zweisprachiger Theaterkurs kann in

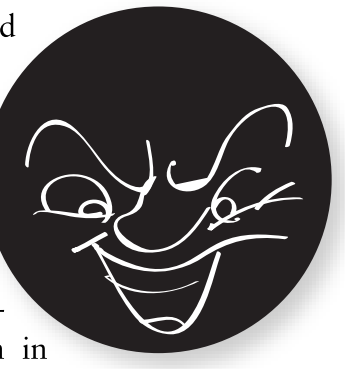

keinem Fall als Konkurrenz (sondern lediglich als Ergänzung) zu einem didaktisierten Deutschunterricht gesehen werden.

\section{Bibliographie}

Benson, P. (2001), Teaching and Researching Autonomy in Language Learning, Longman, Harlow.

Bernhard, T. (2005), „Die Jagdgesellschaft", in: Stücke 1, Suhrkamp, Frankfurt/Main ${ }^{9}$ : 171-249.

Byram, M. (1999), „Acquiring Intercultural Communicative Competence: Fieldwork and Experiential Learning”, in: Bredalla, L. u. Delanoy, W. (Hrsg.), Interkultureller Fremdsprachenunterricht, Narr, Tübingen: 358-380.

Elias, N. (1976), Über den Prozeß der Zivilisation. Soziogenetische und psychogenetische Untersuchungen, Suhrkamp, Frankfurt/Main.

Hallet, W. (2010), Performative
Kompetenz und Fremdsprachenunterricht, Scenario [Online] 1 [Online unter:

http://publish.ucc.ie/scenario/2010/01/hallet/02/de am 26.10.2011].

McDonell, W. (1992), "Language and Cognitive Development Through Cooperative Group

Work", in: Kessler, C. (Hrsg.),

Cooperative Language Learning

- A teacher's resource book, Prentice Hall, Englewood Cliffs: 51-64.

Münster, M. (2008), „Plädoyer für ein zweisprachiges Dramatisierungsexperiment Thomas Bernhards Die Jagdgesellschaft - basiert auf einer Rhythmus orientierten Analyse “, in: Anuario de Estudios Filológicos 31: 133-142. Schewe, M. (2007), Drama und
Theater in der Fremd- und Zweitsprachenlehre - Blick zurück nach vorn, in: Scenario [online], 1 [Online unter http://publish.us.ie/journals/scenario/2007/01/schewe/08/de am 25.10.2011].

Schwerdtfeger, I. (2000), "Leiblichkeit und Grammatik”, in: Düwell, H., Gnutzmann, C. u. Königs, F. (Hrsg.), Fremdsprache in Lehre und Forschung: Dimensionen der didaktischen Grammatik. Festschrift für Günther Zimmermann zum 65. Geburtstag, AKSVerlag, Bochum: 281-303.

1. Eine auf Personen beschränkte Sprachzuteilung wurde für das Projekt Kleider machen Leute (an der Universi- dad de Extremadura 2008/09) durchgeführt. Diese Konzeption beruht auf dem Missverständnis zwischen Protagonisten und den anderen Personen, die in dem Schneidergesellen einen Grafen sehen (wollen). Das Missverständnis beruht auf einer fehlerhaften Kommunikation, die sich das Inszenierungskonzept zu Nutzen macht, insofern der Protagonist Sprache $A$ (Spanisch) und die anderen Personen Sprache $B$ (Deutsch) sprechen. Die mitgeteilte Reflexion der Figuren in einer Sprache (B) und des Protagonisten in einer anderen Sprache (A) über das auf der Bühne Dargestellte ermöglicht es dem Publikum je nach sprachlicher Kompetenz, den Inhalt mindestens auf einer der beiden Sprachen verfolgen zu können. 